\title{
Anatomical variation of co-existing bilaminar tensor of the vastus intermedius muscle and new type of sixth head of the quadriceps femoris
}

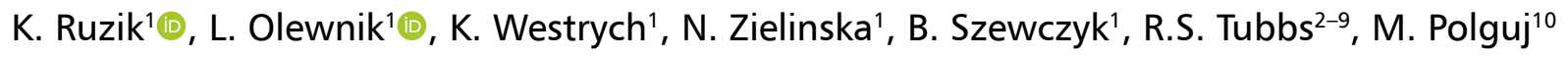 \\ ${ }^{1}$ Department of Anatomical Dissection and Donation, Medical University of Lodz, Poland \\ 2Department of Neurosurgery, Tulane University School of Medicine, New Orleans, LA, United States \\ ${ }^{3}$ Department of Neurology, Tulane Centre for Clinical Neurosciences, Tulane University School of Medicine, \\ New Orleans, LA, United States \\ ${ }^{4}$ Department of Structural and Cellular Biology, Tulane University School of Medicine, New Orleans, LA, United States \\ ${ }^{5}$ Department of Neurosurgery and Ochsner Neuroscience Institute, Ochsner Health System, New Orleans, LA, \\ United States \\ ${ }^{6}$ Department of Anatomical Sciences, St. George's University, St. George's, Grenada \\ 'Division of Gross and Clinical Anatomy, Department of Anatomy, Kurume University School of Medicine, Kurume, \\ Fukuoka, Japan \\ ${ }^{8}$ Department of Surgery, Tulane University School of Medicine, New Orleans, LA, United States \\ ${ }^{9}$ University of Queensland, Brisbane, Australia \\ ${ }^{10}$ Department of Normal and Clinical Anatomy, Chair of Anatomy and Histology, Medical University of Lodz, Poland
}

[Received: 30 April 2021; Accepted: 16 August 2021; Early publication date: 28 September 2021]

\begin{abstract}
Background: We present a case report of quadriceps femoris (QF) with co-existing bilaminar tensor of the vastus intermedius (TVI) muscle and new type of sixth head. Materials and methods: Cadaveric dissection of left thigh of a 72-year-old man was performed for research and teaching purposes at the Department of Anatomical Dissection and Donation, Medical University of Lodz. The left lower limb was dissected using standard techniques according to a strictly specified protocol. Each head of the muscle was photographed and subjected to further measurement. Results: During dissection, an unusual type of TVI muscle was observed. It consisted of two surfaces, superficial and deep. In addition, sixth head of QF muscle grew out from the vastus medialis muscle.

Conclusions: The knowledge of the existence and possible variations of additional heads of QF muscle is necessary during diagnostic process of muscle strains. Moreover, according to course of tendons that heads may take part in patella stabilisation. (Folia Morphol 2022; 81, 4: 1082-1086)
\end{abstract}

Key words: quadriceps femoris, tensor vastus intermedius, anatomical variations, knee joint

\section{INTRODUCTION}

The anatomy of the thigh area and knee joint is highly variable in respect of the ligaments, muscles and vessels around the knee. Interestingly, recent anatomical studies have shed light on the variability of muscles that have long seemed to have constant anatomical structures $[1,18-20,22]$.

Address for correspondence: K. Ruzik, MD, Department of Anatomical Dissection and Donation, Medical University of Lodz, ul. Żeligowskiego 7/9, 90-752 Łódź, Poland, e-mail: kacper.ruzik@umed.lodz.pl

This article is available in open access under Creative Common Attribution-Non-Commercial-No Derivatives 4.0 International (CC BY-NC-ND 4.0) license, allowing to download articles and share them with others as long as they credit the authors and the publisher, but without permission to change them in any way or use them commercially. 
The quadriceps femoris (QF) and patellar ligament $(\mathrm{PL})$ are parts of the knee extensor mechanism; the QF is also a patella and knee joint stabilizer [6, 27]. It is the largest muscle in the body by muscle mass and is traditionally described as consisting of four heads: rectus femoris (RF), vastus medialis (VM), vastus lateralis $(\mathrm{VL})$, and vastus intermedius (VI) $[6,7]$. They all form a common tendon attaching to the base of the patella [11]. Most studies of the anatomical variability of the QF concern duplication of the VM or VL muscle [17]. Golland and Mahon [8] and Holyoke [13] were among the first to report an additional head of the QF. Current anatomical research labels the fifth head of the QF as the "tensor of the vastus intermedius" (TVI) [9]. The incidence of the TVI ranges from $29 \%$ to $100 \%[3,9,28]$.

Grob et al. [9-11] described the TVI muscle with constant origin between the intertrochanteric line and greater trochanter and based their classification on the ability to separate the TVI tendon from the VM and VI muscles. Similar classification was proposed by Veeramani and Gnanasekaran [26] in South Indian population. Olewnik et al. [21] proposed a classification determined by the variability within the proximal attachment and listed three types: TVI independent; TVI grows out from other muscles; and multiple supplementary heads. Both groups also reported the possibility of more additional heads; however, there is no description of the bilaminar variability of the TVI muscle.

This paper describes an extremely rare variant of the TVI, which consists of two layers, superficial and deep. The rare case described can be used to supplement the currently prevailing classification by Olewnik et al. [21].

\section{CASE REPORT}

A 72-year-old male cadaver was subjected to routine anatomical dissection at the Department of Anatomical Dissection and Donation, Medical University of Lodz, Poland. The purpose of the dissection was research and the education of medical students. The left lower limb (hip joint, thigh and knee joint) was dissected using standard techniques according to a specified protocol. The lower limb was positioned in supine position on the dissection table. Dissection began with hip joint capsule resection. Following this, all femoral nerve branches and quadriceps femoris vessels were dissected. The next step was to remove the quadriceps femoris with additional heads, which was the objective of our study. Each head of the vas-

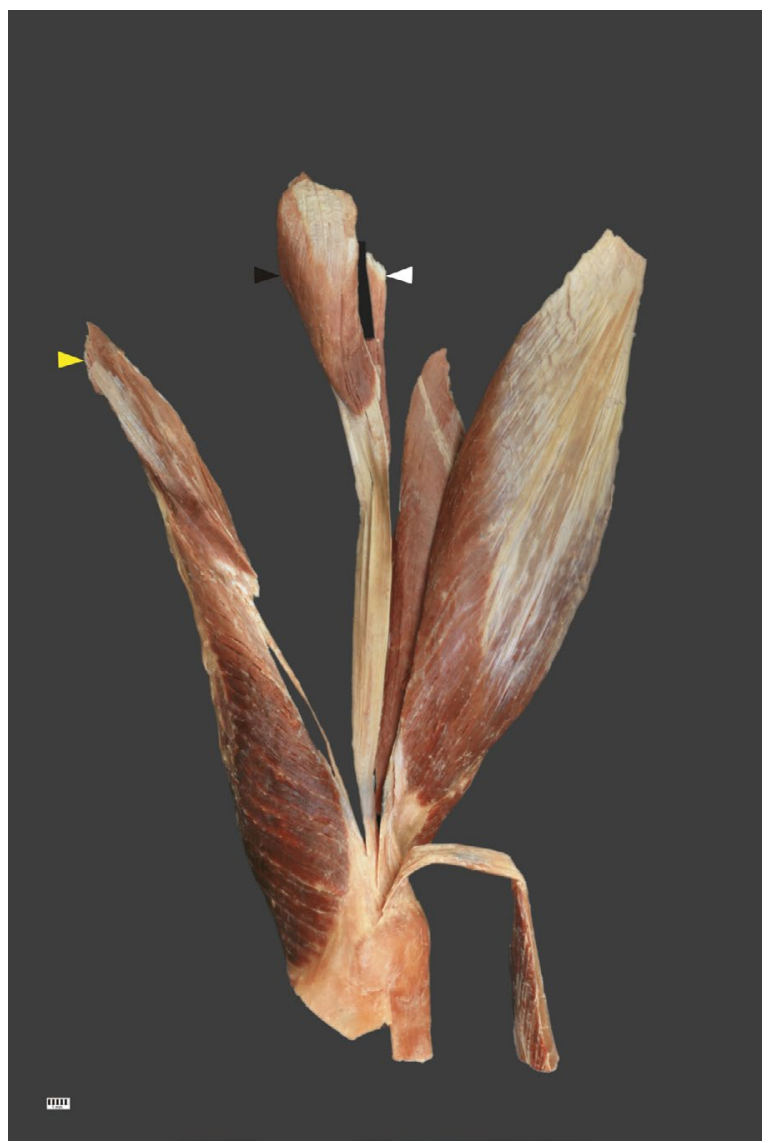

Figure 1. Dissected quadriceps femoris (QF) with additional heads. Black arrow - superficial portion of tensor of the vastus intermedius (TVI) muscle, white arrow - deep portion of TVI muscle, yellow arrow - sixth head of $\mathrm{OF}$.

$\mathrm{ti}$, rectus femoris, tensor of vastus intermedius and sixth muscle belly was separately vascularised from transverse branches of the lateral circumflex femoral artery and innervated with branches originating from the femoral nerve. Finally, the tendons were dissected to identify potential additional tendon bands.

Each head of the muscle was photographed and subjected to further measurement using an electronic calliper (Mitutoyo Corporation, Kawasaki-shi, Kanagawa, Japan), each measurement being performed twice by two researchers with accuracy up to $0.1 \mathrm{~mm}$.

During dissection, an unusual type of TVI muscle was observed. It consisted of two surfaces, superficial and deep. An additional sixth head was visualised, which has no equivalent in either of the classifications of additional QF muscle bellies. This additional head grew out from the VM muscle (Figs. 1, 2). This type of insertion constitutes an extension of type 2 in the Olewnik et al. [21] classification. Measurements of the muscles are given in Table 1. 


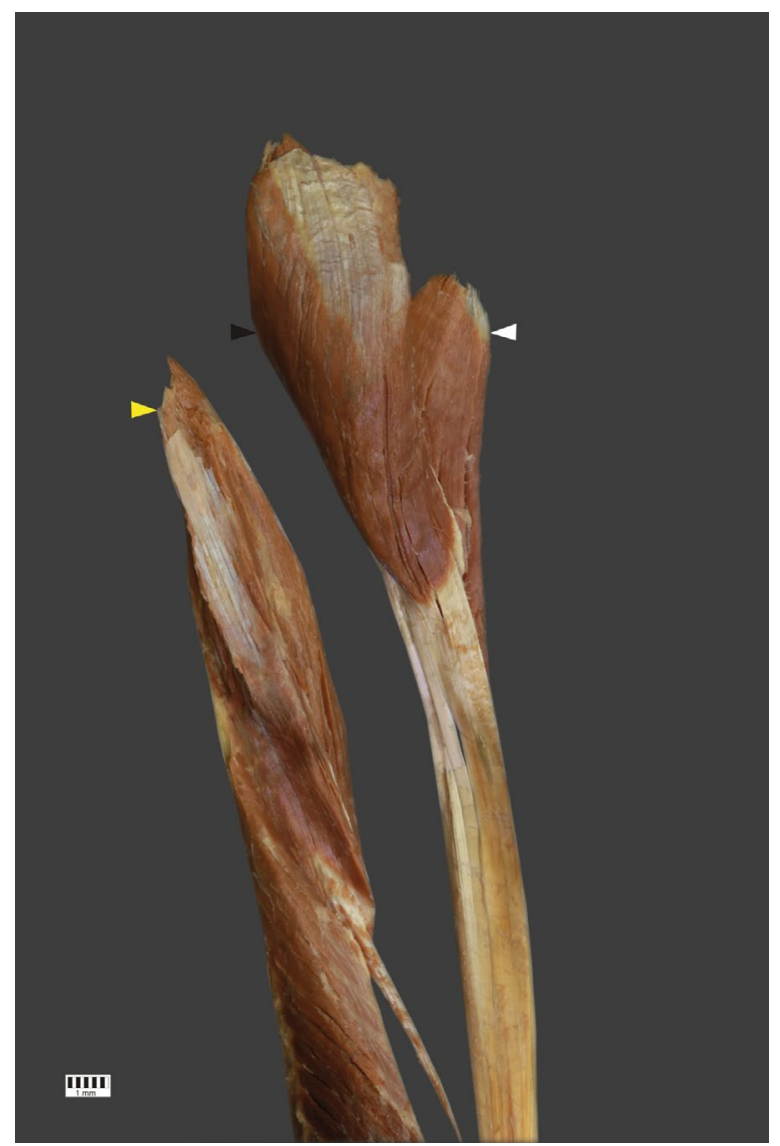

Figure 2. Additional heads of quadriceps femoris (OF) muscle. Black arrow - superficial portion of tensor of the vastus intermedius (TVI) muscle, white arrow - deep portion of TVI muscle, yellow arrow - sixth head of $\mathrm{OF}$.

\section{DISCUSSION}

The muscles of the lower limb derive embryologically from the condensed mesenchyme of the pre-muscle sheath. By the end of fourth week of embryo development there are three regions: thigh, leg, and foot. The QF nucleus expands as a single mass overlying the anterolateral aspect of the middle of the femur's shaft [16]. Afterwards, the mass is apportioned into RF, VL, VM and VI. Division is completed when the embryo is $20 \mathrm{~mm}$ long. The additional head of the QF presumably forms as an extra division of the mesenchyme when the embryo is $11-19 \mathrm{~mm}$ long. However, an embryological study is needed to confirm this [2].

The QF was long considered to have a constant four-headed architecture [6]. The only variations reported concerned duplication of the VM or VL muscle [17]. Some authors have proposed segmental classification of the VM and VL architecture. The VL muscle has three components, superficial, intermediate, and
Table 1. Measurements of the tensor of the vastus intermedius (TVI) muscle and sixth head of quadriceps femoris (QF)

\begin{tabular}{lccc}
\hline & $\begin{array}{c}\text { Superficial } \\
\text { portion of } \\
\text { TVI muscle }\end{array}$ & $\begin{array}{c}\text { Deep } \\
\text { portion of } \\
\text { TVI muscle }\end{array}$ & $\begin{array}{c}\text { Sixth } \\
\text { head of } \\
\text { OF }\end{array}$ \\
\hline Length of the muscle belly & $105.57 \mathrm{~mm}$ & $84.52 \mathrm{~mm}$ & $129.38 \mathrm{~mm}$ \\
Width at the origin & $22.92 \mathrm{~mm}$ & $13.42 \mathrm{~mm}$ & $26.17 \mathrm{~mm}$ \\
Thickness at the origin & $3.04 \mathrm{~mm}$ & $2.02 \mathrm{~mm}$ & $1.27 \mathrm{~mm}$ \\
Width upon passing & $14.07 \mathrm{~mm}$ & $12.20 \mathrm{~mm}$ & $5.68 \mathrm{~mm}$ \\
the muscle belly & & & \\
Thickness upon passing & $1.75 \mathrm{~mm}$ & $1.70 \mathrm{~mm}$ & $1.78 \mathrm{~mm}$ \\
the muscle belly & & & \\
Length of the tendon & $\begin{array}{c}8.55 \mathrm{~mm}, \\
\text { then formed }\end{array}$ & $212.02 \mathrm{~mm}$, & $147.15 \mathrm{~mm}$ \\
& common \\
tendon & \\
& $\begin{array}{c}\text { tendon with } \\
\text { deep portion }\end{array}$ & \\
Width at the insertion & \multicolumn{2}{c}{$8.18 \mathrm{~mm}$} & $9.07 \mathrm{~mm}$ \\
Thickness at the insertion & \multicolumn{2}{c}{$0.77 \mathrm{~mm}$} & $0.82 \mathrm{~mm}$ \\
\hline
\end{tabular}

deep, while the VM muscle consists of longus and obliquus partitions [4, 25].

Golland and Mahon [8] were the first to report an additional head of the QF between the VL and VI muscles. Holyoke [13] described that structure as deviating from normal anatomy. Willan et al. [28] were the next researchers to study an extra muscle belly. However, this additional QF belly long remained without proper nomenclature and classification. Grob et al. [9-11] named the QF head the TVI and created the first fivefold classification based on the course of the tendon. They also reported another additional head that formed a common tendon with the TVI muscle [11]. Veeramani and Gnanasekaran [26] proposed classification based on origin, middle, and distal course of TVI muscle on South Indian population. Olewnik et al. [21] suggested a different anatomical classification based on 106 lower limbs. They proposed three types of TVI muscle with subtypes distinguished by the origin of the additional head. Ruzik et al. [24] were the first to observe additional heads that formed two independent tendons with insertion into the base of the patella. However, it is still unclear whether the TVI muscle is an anatomical variant or a constant component of the QF muscle. In cadaveric studies, the frequency of the additional head ranges from $29 \%$ to $100 \%[3,9,28]$.

The TVI muscle and additional heads as parts of the QF cause extension of the knee joint. The whole muscle has a key role in human locomotion $[6,27]$. The QF also assists in patella stabilisation. Patella 
instability is common problem, especially among young active patients; it results from failure of the medial stabilizers of the patellofemoral joint [15]. The medial patella stabilisation complex is composed of the medial patellofemoral ligament (MPFL), medial patellotibial ligament (MPTL) and medial patellomeniscal ligament (MPML) [12]. Although the QF is a secondary stabilizer, the MPFL is connected to the aponeurosis of the vastus medialis obliquus fibres, creating a dynamic system that stabilizes the patella in the trochlear groove during active flexion. The TVI tendon and the tendon of the additional head insert into the medial part of the patellar base. Owing to the course of that tendon, the additional heads could possibly prevent patella dislocation [11]. It is highly feasible that the bilaminar structure and connection of the sixth head to the VM muscle affects the medialisation of the patella more than other types previously described $[9,21]$. Proper knowledge of the anterolateral thigh anatomy is also necessary in reconstruction surgery. Anterolateral thigh flaps are widely used for reconstruction skin, fascia and muscle of the neck head and after breast mastectomy [7].

Restoring the strength and function of the QF is a key point of rehabilitation after many surgical procedures such as anterior cruciate ligament reconstruction. It should be performed before surgery because it improves the chances of returning to sport. The presence of a TVI or additional head improves the strength of knee extension, especially when it comprises two layers. In addition, the oblique course of the tendon and the connection between the sixth head and the VM muscle decreases the difference of strength between the medial and lateral components of the QF muscle, which can be useful during a rehabilitation protocol.

The RF muscle is the most frequently injured part of the QF [5]. Injury is usually connected to eccentric contraction of the QF muscle. Interestingly, there can be an eccentric contraction in the TVI muscle or other additional head. Owing to the similarity of the courses of the RF and TVI, awareness of the TVI or other additional heads could lead to missdiagnosis. A patient suffering from TVI or additional tendon rupture can present with indefinite knee pain. Nevertheless, there is only one case report of isolated TVI rupture identified by magnetic resonance imaging [10]. Ultrasonography and magnetic resonance imaging of the lower part of the thigh can reveal a tendon rupture. According to Rajasekaran and Hall [23], the transverse plane in a sonographic evaluation can feasibly lead to identifying a TVI rupture.

\section{CONCLUSIONS}

We have reported another anatomical variability of the QF muscle, which should be included in classification. Knowledge of the true anatomy of a QF is essential during diagnosis and treatment of patients with symptoms in this region of the lower limb.

\section{Ethical approval and declarations}

This article describes no studies involving human participants or animals performed by any of the authors.

The Bioethics Committee of the Medical University of Lodz (resolution RNN/114/19/KE) accepted the study protocol. The cadavers were the property of the Department of Anatomical Dissection and Donation, Medical University of Lodz, Poland.

The cadavers belong to the Department of Normal and Clinical Anatomy of the Medical University of Lodz.

\section{Acknowledgements}

The authors sincerely thank those who donated their bodies to science so that anatomical research could be performed. Results from such research can potentially increase mankind's overall knowledge and thus improve patient care. Therefore, these donors and their families deserve our highest gratitude [14].

\section{Conflict of interest: None declared}

\section{REFERENCES}

1. Aragonés $P$, Rodríguez-Niedenführ $M$, Quinones $S$, et al. Popliteal artery: Anatomical study and review of the literature. Ann Anat. 2021; 234: 151654, doi: 10.1016/j. aanat.2020.151654, indexed in Pubmed: 33278577.

2. Bardeen CR. Develoment and variation of the nerves and the musculature of the inferior extremity and the neighboring regions of the trunk in man. Am J Anat. 1907; 6: 23-56.

3. Bonnechère B, Louryan S, Feipel V. Triceps, quadriceps or pentaceps femoris? Need for proper muscle definition. Morphologie. 2020; 104(345): 77-84, doi: 10.1016/j. morpho.2019.06.001, indexed in Pubmed: 31296371.

4. Castanov V, Hassan SA, Shakeri S, et al. Muscle architecture of vastus medialis obliquus and longus and its functional implications: A three-dimensional investigation. Clin Anat. 2019; 32(4): 515-523, doi: 10.1002/ca.23344, indexed in Pubmed: 30701597.

5. Cross TM, Gibbs N, Houang MT, et al. Acute quadriceps muscle strains: magnetic resonance imaging features and prognosis. Am J Sports Med. 2004; 32(3): 710-719, 
doi: $10.1177 / 0363546503261734$, indexed in Pubmed: 15090389.

6. Flandry F, Hommel G. Normal anatomy and biomechanics of the knee. Sports Med Arthrosc Rev. 2011; 19(2): 82-92, doi: 10.1097/JSA.0b013e318210c0aa, indexed in Pubmed: 21540705.

7. Franchi T. Tensor vastus intermedius: a review of its discovery, morphology and clinical importance. Folia Morphol. 2021; 80(4): 792-798, doi: 10.5603/FM.a2020.0123, indexed in Pubmed: 33084009.

8. Golland J, Mahon M. Anatomical variations in human quadriceps femoris muscle. J Anat. 1986: 263-264.

9. Grob K, Ackland T, Kuster MS, et al. A newly discovered muscle: The tensor of the vastus intermedius. Clin Anat. 2016; 29(2): 256-263, doi: 10.1002/ca.22680, indexed in Pubmed: 26732825.

10. Grob K, Fretz Ch, Kuster MS, et al. Knee pain associated with rupture of tensor vastus intermedius, a newly discovered muscle: a case report. J Clin Case Rep. 2016; 6(7), doi: 10.4172/2165-7920.1000828.

11. Grob K, Manestar M, Filgueira L, et al. New insight in the architecture of the quadriceps tendon. J Exp Orthop. 2016; 3(1): 32, doi: 10.1186/s40634-016-0068-y, indexed in Pubmed: 27813020.

12. Hinckel BB, Gobbi RG, Kaleka CC, et al. Medial patellotibial ligament and medial patellomeniscal ligament: anatomy, imaging, biomechanics, and clinical review. Knee Surg Sports Traumatol Arthrosc. 2018; 26(3): 685-696, doi: 10.1007/s00167-017-4469-y, indexed in Pubmed: 28289819.

13. Holyoke A. An unusual variation in quadriceps femoris. J Anat. 1987; 227.

14. Iwanaga J, Singh V, Ohtsuka A, et al. Acknowledging the use of human cadaveric tissues in research papers: Recommendations from anatomical journal editors. Clin Anat. 2021; 34(1): 2-4, doi: 10.1002/ca.23671, indexed in Pubmed: 32808702.

15. Krebs C, Tranovich M, Andrews K, et al. The medial patellofemoral ligament: Review of the literature. J Orthop. 2018; 15(2): 596-599, doi: 10.1016/j.jor.2018.05.004, indexed in Pubmed: 29881201.

16. Lewis WH. The development of the muscular system. Lewis WH (1910) The development of the muscular system. In: Keibel F MF (eds). Man Hum Embryol. 1st edn. JB Lippincott Company, Philadelphia 1910: 454-522.
17. MacAlister A. Observations on the mucular variations in the human anatomy. Third series with a catalogue of the principal muscular variations hitherto published. TransRoy IrishAcad Sci. 1875: 1-130.

18. Olewnik $k$, Gonera B, Kurtys K, et al. The anterolateral ligament of the knee: a proposed classification system. Clin Anat. 2018; 31(7): 966-973, doi: 10.1002/ca.23267, indexed in Pubmed: 30144325.

19. Olewnik $Ł$, Kurtys K, Gonera B, et al. Proposal for a new classification of plantaris muscle origin and its potential effect on the knee joint. Ann Anat. 2020; 231: 151506, doi: 10.1016/j. aanat.2020.151506, indexed in Pubmed: 32173563.

20. Olewnik $t$, Łabętowicz $P$, Podgórski $M$, et al. Variations in terminal branches of the popliteal artery: cadaveric study. Surg Radiol Anat. 2019; 41(12): 1473-1482, doi: 10.1007/ s00276-019-02262-3, indexed in Pubmed: 31134299.

21. Olewnik Ł, Tubbs RS, Ruzik K, et al. Quadriceps or multiceps femoris? Cadaveric study. Clin Anat. 2021; 34(1): 71-81, doi: 10.1002/ca.23646, indexed in Pubmed: 32644202.

22. Olewnik $t$, Zielinska N, Paulsen $F$, et al. A proposal for a new classification of soleus muscle morphology. Ann Anat. 2020; 232: 151584, doi: 10.1016/j.aanat.2020.151584, indexed in Pubmed: 32810614.

23. Rajasekaran S, Hall MM. Sonographic appearance of the tensor of the vastus intermedius. PM R. 2016; 8(10): 1020-1023, doi: 10.1016/j.pmrj.2016.04.002, indexed in Pubmed: 27108157.

24. Ruzik K, Waśniewska A, Olewnik $t$, et al. Unusual case report of seven-headed quadriceps femoris muscle. Surg Radiol Anat. 2020; 42(10): 1225-1229, doi: 10.1007/ s00276-020-02472-0, indexed in Pubmed: 32318799.

25. Toia F, D'Arpa S, Brenner E, et al. Segmental anatomy of the vastus lateralis. Plast Reconstr Surg. 2015; 135(1): 185e-198e, doi: 10.1097/prs.0000000000000842.

26. Veeramani R, Gnanasekaran D. Morphometric study of tensor of vastus intermedius in South Indian population. Anat Cell Biol. 2017; 50(1): 7-11, doi: 10.5115/ acb.2017.50.1.7, indexed in Pubmed: 28417049.

27. Waligora AC, Johanson NA, Hirsch BE. Clinical anatomy of the quadriceps femoris and extensor apparatus of the knee. Clin Orthop Relat Res. 2009; 467(12): 3297-3306, doi: 10.1007/ s11999-009-1052-y, indexed in Pubmed: 19690926.

28. Willan PL, Mahon M, Golland JA. Morphological variations of the human vastus lateralis muscle. J Anat. 1990; 168: 235-239, indexed in Pubmed: 2323995. 